\title{
Revuelta social y proceso \\ constituyente en Chile
}

\section{Social Revolt and the Constituent \\ Process in Chile}

Claudia Heiss ${ }^{1}$

Cómo citar este artículo: Heiss, C. (2021). Revuelta social y proceso constituyente en Chile. Revista de Ciencias Sociales Ambos Mundos, (2), 69-78. https://doi.org/10.14198/ambos.20981

\section{Resumen}

En octubre de 2019 se produjo en Chile la mayor movilización social desde el retorno a la democracia, en 1990. Precedido de más de una década de acción colectiva contenciosa con diversas agendas, este estallido o revuelta impulsó transformaciones en materia de derechos, protección social y nuevas reglas de la política. La más comprehensiva de ellas es la creación de una nueva constitución que reemplace la carta fundamental impuesta por la dictadura militar. El artículo analiza dos elementos del proceso constituyente que busca dar una salida institucional a la grave crisis social y política expresada en la revuelta que comenzó en 2019: el papel que jugó la rigidez institucional, protectora de las normas impuestas por la dictadura, y la crisis de la representación política que ha afectado al país.

Palabras claves: Elaboración constitucional; política chilena; movimientos sociales.

\section{Abstract}

In October 2019, Chile experienced the largest social mobilization since the return to democracy, in 1990. Following over a decade of contentious collective action addressed at several issues, this outbreak or revolt advocated transformations in the areas of rights, social protection and the rules for political decision-making. The most comprehensive of these changes is the creation of a new constitution aimed at replacing the one imposed by the military dictatorship. The article analyzes two elements of the constituent process that seeks to institutionally channel the social and political crisis expressed in the revolt that began in 2019: the role played by an institutional rigidity protective of the norms imposed by the dictatorship, and the crisis of political representation in the country.

Keywords: Constitution-making; Chilean politics; Social movements.

1. Claudia Heiss, Universidad de Chile, Chile. cheiss@iap.uchile.cl https://orcid.org/0000-0002-2690-7175 


\section{INTRODUCCIÓN ${ }^{1}$}

El viernes 18 de octubre de 2019 se desató en Chile una masiva movilización social que incluyó, en las semanas siguientes, protestas callejeras, acciones de desobediencia civil y actos de violencia. La academia y la opinión pública iniciaron entonces un debate sobre cómo entender, y en consecuencia cómo nombrar, estos acontecimientos. Desde el mismo día 18 se empezó a usar la expresión "estallido social", sugiriendo un episodio puntual de liberación de una gran fuerza contenida. Más tarde comenzó a extenderse el uso del término "revuelta", apelando a una movilización de carácter estructural o de ribetes casi revolucionarios.

El estallido o revuelta no fue orquestado por ningún grupo o sector identificable, ni tenía la pretensión de hacerse con el poder, como ocurre con las revoluciones. Sin embargo, sí apuntaba a dos adversarios más o menos determinados: el modelo socioeconómico y el sistema político que han imperado en Chile desde el fin de la dictadura militar en 1990 (Garretón, 2021; Somma et. al., 2020). En este sentido, es relevante identificar el nivel de continuidad o de ruptura que tiene el estallido o la revuelta con procesos de larga data como el aumento de la política contenciosa y la aparición de nuevos movimientos sociales desde la década de 2000 , la fuerte y sostenida caída en la participación electoral desde 1990, la crisis de legitimidad de instituciones políticas y sociales, y la desaceleración del crecimiento económico durante la última década, entre otros.

En lo que sigue, se analizan dos aspectos que marcan el proceso constituyente con el que Chile busca dar una salida institucional a la grave crisis social y política que se expresó en la revuelta que comenzó en 2019. Primero, se aborda el papel de la rigidez institucional que por décadas protegió de reformas a normas impuestas por la dictadura. Luego se analiza la crisis de la representación política que ha afectado al país. Por último, se esbozan algunas conclusiones sobre este proceso.

1. Algunos contenidos de este artículo, especialmente la segunda parte, se desarrollaron antes en Heiss, C. (2021). "Latin America Erupts: Re-founding Chile". Journal of Democracy, 32(3), 33-47. http://doi. org/10.1353/jod.2021.0032

\section{RIGIDEZ INSTITUCIONAL: PROTECTORA DEL STATU QUO}

La mantención de componentes fundamentales del modelo económico y político de la dictadura a través de gobiernos democráticos fue objeto de cuestionamientos ya en la década de 1990, cuando un grupo de dirigentes al interior de la coalición de centroizquierda Concertación de Partidos por la Democracia elaboró una autocrítica y se declaró "autoflagelante", en contraposición a quienes se declaraban como "autocomplacientes". El sociólogo Tomás Moulian planteó que la Concertación había efectuado una operación de "transformismo", consistente en dotar al sistema neoliberal de un "rostro humano", a fin de consolidar y profundizar el modelo social y económico impuesto por la dictadura (Moulian, 1997).

En el marco del estudio de las políticas de la izquierda en América Latina, un análisis de los gobiernos socialistas de Ricardo Lagos (2000-2006) y la primera administración de Michelle Bachelet (2006-2010), calificó como “moderados" los cambios en políticas laborales y de regulación del mercado, y de "escaso" su fomento de la participación ciudadana. Tres causas explicarían esa moderación: el rol de los liderazgos políticos y su poca vinculación con bases partidistas y de la sociedad civil; el hecho de que se tratara de gobiernos de coalición; y las limitaciones de los legados políticos y económicos de Pinochet. El último punto se refiere especialmente a las limitaciones políticas emanadas del diseño institucional de la dictadura, en especial la Constitución de 1980 (Huber, Pribble y Stephens, 2010, p. 77).

Desde un análisis de la economía política post transicional, Madariaga señala que "Ios pilares básicos de la política económica heredados de la dictadura de Pinochet se mantienen incólumes" (Madariaga, 2019, p. 82). La explicación, según este autor, descansa en tres factores: el predominio de ideas económicas neoliberales, instituciones políticas heredadas de la dictadura e intereses económicos empresariales. En relación con el primer factor, Madariaga señala que la falta de cuestionamientos y propuestas alternativas al modelo económico en el ámbito de las ideas fue el fruto de procesos de socialización impulsados tanto desde la educación como por los medios de comunicación. En relación con las instituciones políticas, destaca la creación de poderes de veto sobre la continuidad o cambio de la política pública, incluyendo "sesgos de representación sistemáticos" 
que favorecieron a quienes apoyan el neoliberalismo. El empresariado, por su parte, habría utilizado su poder económico para promover sus intereses en la decisión política.

Uno de los efectos más notorios del estallido o revuelta de 2019 fue hacer posible un proceso constituyente democrático para reemplazar la Constitución de 1980, impuesta en dictadura por el general Augusto Pinochet. Esta idea, que surgió con claridad como demanda ciudadana al menos desde 2015, no había sido recogida en forma viable por el sistema político. Sin embargo, tras cuatro semanas de protestas y represión policial, el 15 de noviembre de 2019 la mayor parte de los partidos políticos con representación parlamentaria aprobó un acuerdo para dar una salida institucional a la crisis a través de un proceso constituyente.

Si bien la literatura de la ciencia política suele elogiar la solidez de las instituciones, el caso de la Constitución chilena de 1980 se ha convertido en un ejemplo de cómo instituciones rígidas pueden ser perjudiciales para la gobernabilidad y la estabilidad institucional. Murillo, Levitsky y Brinks (2021) señalan que la ciudadanía en Chile identificó la desigualdad económica con la Constitución de 1980, volviéndola blanco de las protestas. Este cuerpo normativo, que sobrevivió por muchos años al régimen que le dio origen, "es un ejemplo claro de una institución fuerte, que logró altos niveles de cumplimiento y cuyas disposiciones centrales resultaron resistentes al cambio" (p. 12). Este hecho, sin embargo, se convirtió en un problema al obstruir la adaptación de la política a las demandas sociales, impidiendo alinear las normas sociales con las instituciones formales. La constitución contribuyó, así, a agudizar una crisis de legitimidad del sistema democrático.

Si bien en 2019 el sistema político logró dar una salida institucional a la crisis social a través del proceso constituyente, es importante destacar que los acuerdos transversales que lo hicieron posible fueron una reacción a la presión de la calle y las organizaciones sociales. De hecho, las propuestas de reemplazo constitucional que se venían discutiendo por décadas fueron principalmente resistidas por las autoridades en el poder, incluida la centro-izquierda. ¿A qué se debió el cambio que llevó a las élites políticas a transitar desde el rechazo a la apertura, como lo expresó el acuerdo del 15 de noviembre? Escudero (2021) sostiene que al menos tres elementos explican esta transformación: primero, un proceso acumulativo de presión social, iniciado con las protestas estudiantiles del año 2006. Segundo, el malestar social vinculado al estallido o revuelta de octubre de 2019, que aumentó los costos para toda la élite política, desde la derecha a la izquierda, de negar la opción de un proceso constituyente. Tercero, la búsqueda de acuerdos políticos cupulares orientados a reducir la incertidumbre en el proceso de elaboración de la nueva constitución (Escudero, 2021). Este último punto tuvo especial relevancia en posibilitar que los sectores conservadores accedieran a negociar un proceso constituyente, aún en contra de sus preferencias sustantivas.

Entre el retorno de la democracia en 1990 y la revuelta social de 2019, cinco gobiernos de centroizquierda gobernaron el país durante 24 años. A ellos se suman dos gobiernos de derecha, con un total de 8 años, hasta 2022. El reclamo del movimiento social, resumido en la frase "no son 30 pesos, son 30 años", parece apuntar a la falta de reformas estructurales en las arenas política, social y económica durante esas administraciones. Bajo los gobiernos de coaliciones de centro-izquierda y de derecha, se mantuvieron estables los niveles de desigualdad y el modelo de focalización del gasto social en los grupos más vulnerables, en desmedro de políticas universales de bienestar. En el periodo se agudizó el distanciamiento entre elites y ciudadanía y se generó un fuerte rechazo a los partidos políticos, con independencia de su posición ideológica.

El Informe de Desarrollo Humano 2015 del Programa de Naciones Unidas para el Desarrollo (PNUD) en Chile describió con claridad una gran brecha en las percepciones y opiniones entre élites y ciudadanía, advirtiendo una preocupante incapacidad de las instituciones para canalizar demandas sociales. Luna y Altman (2011) criticaron el hasta entonces admirado sistema de partidos chileno, argumentando que se encontraba "desarraigado pero estable", es decir, aunque los partidos políticos parecían programáticos y constantes, habían perdido conexión con las bases ciudadanas $y$, por lo tanto, la capacidad de generar espacios legitimados de representación democrática. A esto se sumaron varios indicadores de una dramática caída en la confianza en las instituciones sociales y políticas, incluidos los partidos políticos, el congreso, la Iglesia católica y la policía (Segovia, 2015).

La rigidez de instituciones diseñadas en dictadura es una variable relevante para explicar la crisis de la representación política y el debilitamiento de la democracia chilena. El objetivo de estas normas era proyectar el statu quo del periodo 
autoritario en la naciente democracia, fenómeno conocido como "enclaves" de un régimen en su sucesor (Garretón, 2003). Lo que la dictadura buscaba proteger de futuras reformas democráticas era, por una parte, un papel preferente del mercado en la provisión de servicios públicos como educación, salud y pensiones. Por otra parte, se buscaba generar un sistema capaz de aislar la decisión política de presiones sociales que los militares y sus aliados civiles percibían como potencialmente destructivas de la libertad económica. Fue esa concepción acerca de los peligros de las propias fuerzas internas de la democracia la que llevó a acuñar la idea de una "democracia protegida" (Huneeus, 2016; Madariaga, 2019).

El objetivo de restringir el papel del estado en la economía se plasmó en el concepto de "estado subsidiario", implícito en la constitución. Por ese concepto se entendía el predominio de soluciones de mercado a problemas públicos, y el resguardo constitucional de la libertad de elegir entre prestaciones sociales públicas o privadas, antes que del derecho a recibirlas. El supuesto objetivo de evitar monopolios estatales se convirtió, en la práctica, en garantizar a los privados un papel preferencial. Según esta concepción, sólo allí donde el mercado no puede o no tiene interés en intervenir, puede hacerlo el estado.

La transición a la democracia se inició en 1990 por los recovecos que dejaron los "enclaves" de la dictadura (Garretón, 2003), lo que significó mantener a Pinochet como comandante en jefe del Ejército hasta 1998 y luego como senador vitalicio hasta su arresto en Londres en octubre de ese año por crímenes de lesa humanidad. La existencia de senadores designados y la incapacidad del presidente para destituir a los comandantes en jefe de las Fuerzas Armadas permanecieron vigentes hasta la reforma constitucional de 2005, quince años después del retorno de la democracia.

El problema constitucional chileno no fue superado por la vía de reformas. A pesar de importantes modificaciones al texto, como aquellas aprobadas en 1989 y 2005, la "incomodidad constitucional" (Busch, 2012) y la percepción de que se trataba de una "constitución tramposa" (Atria, 2013) aumentaron junto con las demandas sociales y las movilizaciones. Producto del poder de veto de la derecha, la constitución mantuvo su sección dogmática casi intacta, con fundamentos filosóficos incompatibles con un estado constitucional verdaderamente democrático y manteniendo el carácter neoliberal del orden económico-social.
El malestar con la Constitución de 1980 se explica, según Tania Busch, por una combinación de tres elementos principales: su origen autoritario e ilegítimo; la consagración de un régimen poco democrático, heredero de la noción de democracia protegida o tutelada que buscó imponer la dictadura, y el establecimiento de un sistema neoliberal deficitario en materia de derechos económicos y sociales. La ilegitimidad de origen es especialmente evidente en los capítulos relativos a las bases de la institucionalidad y los derechos fundamentales, que obedecen a una concepción particular de los derechos promovida por la junta militar y sus partidarios. Fernando Atria (2013), por su parte, destaca cuatro "trampas" o "cerrojos" que han redundado en que la constitución actúe como un impedimento al cambio político: el sistema electoral binominal para la conformación del congreso; los altos quórums de reforma constitucional de 2/3 o 3/5 según la materia de que se trate; el papel del Tribunal Constitucional como guardián de la constitución; y las leyes orgánicas constitucionales, que exigen $4 / 7$ de los votos para su modificación.

\subsection{Sistema electoral binominal}

El sistema electoral binominal, para la elección del Senado y la Cámara de Diputados, se caracterizó por elegir a dos representantes por cada unidad territorial, llamada circunscripción, en el caso del Senado, y distrito en la Cámara de Diputados. Si bien se trata de un sistema proporcional, y no mayoritario, ha sido calificado como el menos proporcional de los sistemas pertenecientes a esta categoría. Su mayor beneficiario, en términos de votos, fue la primera minoría, es decir, la coalición de derecha. Bastaba que ese sector obtuviera un tercio más uno de los votos en un distrito o circunscripción para asegurar la elección de al menos uno de los dos escaños en disputa. Así, el sistema electoral garantizaba a la minoría una representación igual o casi igual a la de la mayoría.

El sistema binomial creó incentivos para la formación de dos coaliciones electorales. Cada unidad territorial elegía a dos representantes al congreso, pero para que esos dos escaños pertenecieran a la misma coalición era necesario que una lista duplicara a la otra. La consecuencia de esta fórmula fue que, con un poco más de $1 / 3$ de los votos, una coalición obtenía la mitad de los escaños. Como resultado, la composición del congreso sobrerrepresentó por décadas a la minoría. 
Además, el sistema hacía casi imposible la competencia de terceras fuerzas políticas. A pesar de que la Concertación ganó regularmente las elecciones parlamentarias, el sistema electoral impidió que obtuviera las mayorías necesarias para aprobar reformas políticas significativas.

El sistema binominal fue clave para alimentar el descontento con la política electoral. Hizo que los resultados de las elecciones parlamentarias fueran extremadamente predecibles. La elección de los miembros del congreso dependía más de que los candidatos consiguieran la nominación por parte de una coalición que de la votación en las urnas. Este distorsionador mecanismo se abandonó sólo en 2015, 25 años después del retorno de la democracia. En su reemplazo, se instauró un sistema proporcional, con cuotas de género y derecho a voto para chilenos en el extranjero, el que se aplicó por primera vez en las elecciones parlamentarias de 2017.

El sistema electoral binomial se convirtió en un símbolo de la inmutabilidad de las reglas políticas. Además, se vio como una prueba de que las élites de centroizquierda y derecha privilegiaban tomar decisiones entre ellas sobre canalizar demandas sociales, fenómeno conocido como "el duopolio". Este concepto fue más allá de las elecciones para encarnar una "cultura binominal" donde cada decisión requería el acuerdo de ambas partes, en desmedro de la regla de mayorías (Atria 2013; Escudero 2018).

\subsection{Tribunal Constitucional}

Una reforma constitucional redujo, el año 2005, la tutela militar sobre el sistema político modificando la composición y competencias del Consejo de Seguridad Nacional, reformando los estados de excepción y eliminando de la constitución el papel de "garantes" del orden institucional asignado a las Fuerzas Armadas. Sin embargo, estos cambios requerían los votos de la derecha: su moneda de cambio fue fortalecer las atribuciones del Tribunal Constitucional (TC), lo que aumentó la lógica anti mayoritaria y la protección del statu quo por esa vía.

La reforma de 2005 aumentó de 7 a 10 los integrantes del TC. También fortaleció sus facultades, al transferirle desde la Corte Suprema la decisión sobre el recurso de inaplicabilidad, que es la decisión sobre si una norma debe o no aplicarse en un caso particular de conformidad con la constitución. Además, la reforma de 2005 otorgó al TC la nueva atribución de eliminar una ley aprobada por el congreso. Esta atribución se usó, por ejemplo, cuando en 2018 el TC derogó la ley que otorgaba al Servicio Nacional del Consumidor, SERNAC, la capacidad de aplicar sanciones a las empresas que violaran derechos del consumidor. El fallo provocó indignación, a la luz de recientes casos de colusión y prácticas abusivas del comercio. La reforma de 2005 también reforzó la percepción de que los miembros del TC se designan de acuerdo con su lealtad política más que por sus credenciales profesionales. Junto con dictar un número creciente de sentencias, el TC comenzó a recibir críticas por distorsionar la labor legislativa al impedir la promulgación de leyes de alta connotación pública, como si se tratara de una "tercera cámara" legislativa. (Ahumada 2019; Bassa 2015).

\subsection{Quorum de reforma constitucional}

El texto de 1980 exige una votación de 3/5 o 2/3 en el congreso para la reforma constitucional, según la norma de que se trate. Así, por ejemplo, un proyecto de ley que buscaba declarar el agua como bien de uso público en la constitución fue rechazado por el Senado en enero de 2020 a pesar de obtener 24 votos favorables y solo 12 en contra. El quórum de $2 / 3$ exigía 29 votos favorables para cambiar la propiedad privada de los derechos de agua establecidos por la dictadura en la constitución. Como en este ejemplo, una tercera parte de los votos de la derecha ha sido suficiente desde 1990 para evitar cambios en normas establecidas en un contexto autoritario.

\subsection{Leyes orgánicas constitucionales}

Un cuarto elemento de protección del statu quo han sido las Leyes Orgánicas Constitucionales (LOCs), que no existían antes de la Constitución de 1980, y que sólo pueden modificarse con una votación de 4/7 en el congreso. De esta forma, las LOCs extienden el principio contra mayoritario más allá de la constitución, a una forma especial de legislación. Muchas de estas leyes fueron redactadas apresuradamente durante el verano de 1990, sólo unas semanas antes del cambio de gobierno en marzo de ese año, con el evidente objetivo de atar de manos al gobierno civil democrático entrante. El quórum de 4/7 es necesario para aprobar, modificar 0 derogar normas en 18 materias, incluyendo educa- 
ción, elecciones, Banco Central y partidos políticos, entre otros. Estas leyes también están sujetas a un control preventivo obligatorio por parte del TC. EI papel de las LOCs como mecanismo para prevenir reformas políticas se ha visto reforzado tanto por el sistema electoral binomial vigente hasta la elección del congreso que entró en funciones en 2018, como por el control preventivo del TC.

\section{EL COLAPSO DE LA REPRESENTACIÓN INSTITUCIONAL}

El fenómeno de la crisis de representación política, visible en distintas partes del globo desde comienzos del siglo XXI, tiene en el caso chileno ciertas peculiaridades agravantes: una de ellas es la rigidez institucional heredada de la dictadura. La falta de reformas políticas y económicas tras la recuperación democrática se tornó insostenible desde mediados de la década de 2000. El movimiento estudiantil de 2006, que demandaba el fin del lucro y la capacidad de seleccionar al alumnado en escuelas secundarias con financiamiento público, inició un intenso y duradero ciclo de política contenciosa (Donoso y Von Bulow, 2016; Somma et. al., 2020; Garretón 2021).

El objetivo de la movilización de 2006 era modificar la Ley Orgánica Constitucional de Educación. Contribuyó así a vincular demandas sociales con la crítica a las reglas de decisión política heredadas de la dictadura. Protestas masivas volvieron a producirse en 2011, esta vez de la mano del movimiento estudiantil universitario que exigía gratuidad y mejoras en la calidad de la educación, en el contexto de un sistema altamente desregulado y financiado principalmente a través del endeudamiento de las familias en el mercado financiero.

Las agendas de los movimientos sociales incluyeron la protección del medio ambiente, reconocimiento de derechos de los pueblos indígenas, derechos de las disidencias sexuales, movimiento feminista, rechazo al sistema privado de pensiones, mejoras a la salud pública, acceso a la vivienda y descentralización, entre otras (Donoso y Von Bulow, 2016). Tras más de una década de intensa movilización social, el estallido de 2019 pareció condensar esas distintas agendas específicas en una demanda general por "dignidad" y derechos sociales (Garretón, 2021; Somma et. al., 2020). La percepción de abuso por parte de un sistema de mercado desregulado y de un estado que no garantiza derechos sociales se sumó al rechazo a la política institucional, alimentado en parte por la poca relevancia de la política electoral. El estallido se desencadenó, en efecto, luego de tres décadas de disminución sostenida de la participación electoral y la legitimidad de las instituciones políticas. (PNUD, 2017; Segovia, 2015).

La revuelta social y la demanda de cambio institucional tuvieron un componente relevante de rechazo a las élites. Esta desconfianza se alimentó, en parte, de escándalos de corrupción que se conocieron desde 2014 , asociados al financiamiento ilegal de la política y la injerencia de grupos empresariales en la formación de leyes. Varios estudios han mostrado una brecha significativa entre las percepciones y preferencias de las élites políticas, sociales y económicas, por un lado, y las de la ciudadanía, por el otro. El informe del PNUD de 2015 mostró la diferencia entre estos grupos al consultar acerca de la preferencia por una intervención más fuerte del estado en salud, educación, pensiones y explotación del cobre. Entre el $20 \%$ y el $30 \%$ de las élites estuvieron de acuerdo, frente a un $70 \%$ a $80 \%$ de la ciudadanía. De manera similar, un estudio de 2021 realizado por el Centro de Estudios de Conflicto y Cohesión Social (COES) concluyó que el papel social del estado no era relevante para las élites económicas, con solo un $28 \%$ a favor de aumentar la responsabilidad del estado en garantizar el sustento de todas las personas. En cambio, la idea contó con el apoyo del $54 \%$ de las y los encuestados no-élite. Las élites económicas se mostraron a favor de aumentar el número de empresas e industrias en manos privadas (62\%), lejos del público en general (19\%).

La crisis de los partidos políticos como mediadores legítimos entre sociedad y política es un fenómeno general que presenta en Chile ciertas particularidades. Luna (2021) señala que hay al menos tres factores que dificultan hoy en día la función mediadora que jugaron en el pasado los partidos: primero, la compresión de los tiempos de la política, donde adquiere creciente relevancia la inmediatez de las redes sociales y las encuestas; segundo, la fragmentación social y territorial, que atenta contra la identificación con un programas político general y definido; por último, el surgimiento de una ciudadanía "monotemática", que se moviliza por causas específicas pero no por programas políticos comprehensivos o ideológicos. Todos estos factores hacen suponer que será cada vez más difícil la construcción de legitimidad política a través de partidos como los conocimos hasta hoy. 
Junto con esa explicación de carácter general, Barozet (2016) apunta a dos factores que inciden en la brecha entre sociedad y política en Chile: la decisión de los partidos políticos de centro-izquierda al inicio de la transición de desmovilizar a la sociedad civil para garantizar la estabilidad del proceso, y la dificultad de generar una política verdaderamente representativa, en un contexto de aguda desigualdad socioeconómica. El énfasis en acuerdos intra-élites desde el retorno a la democracia, en desmedro de la participación ciudadana, contribuyó a generar una "democracia de baja intensidad" (Barozet, 2016). Chile se encuentra entre los países más desiguales de la OCDE, y la reducción de la desigualdad resultante de impuestos y transferencias es la más baja del grupo, alcanzando solo el $5 \%$ en comparación con un promedio de la OCDE de alrededor del 25\% (Causa y Hermansen, 2019). En este contexto, la percepción de que las élites se encuentran encapsuladas ha provocado un grave problema de gobernabilidad.

La crisis de la representación política, que llegó a arrojar una aprobación de los partidos políticos del $2 \%$ en la encuesta del Centro de Estudios Púbicos de abril 2021, plantea un desafío complejo para el futuro de la democracia chilena. Los canales tradicionales e institucionalizados de la mediación política no parecen estar cumpliendo su función, y sin embargo la democracia representativa no puede prescindir de dicha mediación. Frente a este escenario, surge la pregunta por el papel que podrían jugar por un lado la política no mediada, a través de mecanismos de democracia directa, y eventuales nuevos actores mediadores, distintos de los partidos políticos.

El abrumador triunfo de las candidaturas independientes en las elecciones de mayo de 2021 para integrar la Convención Constitucional da cuenta de la marcada preferencia ciudadana por candidaturas sin militancia partidista. Las listas de independientes, autorizadas por primera vez para competir en listas que les permitieron sumar votos a nivel distrital, algo normalmente reservado sólo a los partidos, obtuvieron 48 de 155 representantes. Adicionalmente, otras 56 candidaturas independientes electas dentro de las listas de partidos llegaron a la asamblea. En total, el $67 \%$ de la convención constitucional no tiene afiliación a ningún partido político.

De las casi 80 listas independientes que compitieron en todo el país, las más organizadas a nivel nacional obtuvieron los mejores resultados: 24 escaños para la Lista de Pueblo, 8 para Movimientos Sociales, 11 para Independientes No Neutrales. Es- tas listas tienen una orientación de izquierda o centro-izquierda y un mensaje anti partidos políticos. Si bien Independientes No Neutrales está compuesta por profesionales asociados a la centro-izquierda, las otras dos tienen un perfil más vinculado a movimientos sociales. Sus plataformas incluyen la protección del medio ambiente, la agenda feminista y el derecho al agua.

El éxito de las listas de independientes plantea la pregunta de cómo mejorar la representatividad del sistema político chileno sin prescindir de proyectos programáticos y de alcance nacional. La discusión apunta a los mecanismos necesarios para democratizar la participación política y la gran interrogante es si los partidos políticos podrán emerger de su lamentable apoyo del $2 \%$. Se trata, en otras palabras, de evitar el elitismo y la falta de nexo territorial que han mostrado los partidos, promoviendo su democratización, pero sin hacerlos desaparecer. De lo contrario, el resultado podría ser una desinstitucionalización completa que deje la política chilena sin canales de intermediación.

La derecha sufrió una importante derrota en la elección a la Convención Constitucional, a pesar de competir en una lista unida, "Vamos por Chile". Esta lista agrupaba a los partidos Renovación Nacional (RN), Unión Democrática Independiente (UDI) y Evolución Política (Evópoli), más el partido Republicano, de extrema derecha. La unidad les garantizó una sobrerrepresentación en la Convención, porque en el sistema proporcional las listas suman internamente sus votos para distribuir los escaños en cada distrito. En contraste, la izquierda tuvo una dispersión en tres listas, además de casi 80 listas de independientes que en su mayoría disputaron el voto de centro-izquierda. Sin embargo, aún con esta sobrerrepresentación, "Vamos por Chile" solo obtuvo 37 escaños, lejos de los 52 necesarios para bloquear propuestas en la Convención.

El partido con más votos en esta lista fue UDI, con 17 convencionales, seguido de RN con 15 y Evópoli con 5. El aumento de la participación de jóvenes de las zonas urbanas pobres, que había sorprendido en el plebiscito de octubre, no ocurrió en esta elección. Sin embargo, una disminución generalizada de la participación y su concentración en las áreas más ricas no impidió que la derecha obtuviera un magro $20 \%$ de la Convención. Esta baja votación puede interpretarse como un castigo al gobierno por su manejo de la revuelta social de 2019 $y$, especialmente, por el manejo de la pandemia. Las autoridades tardaron más de un año en ceder a las presiones para otorgar un ingreso de emergen- 
cia sustantivo, y esto solo luego de que el congreso aprobara el retiro de los fondos privados de pensiones ante una crisis que generó la peor contracción económica en décadas.

Los partidos de centro-izquierda compitieron en la "Lista de Apruebo", que incluía a la Democracia Cristiana (DC), Partido por la Democracia (PPD), Partido Radical (PR), Partido Socialista (PS), Partido Progresista (PRO) y Ciudadanos. El conglomerado obtuvo sólo 25 escaños. Su partido más votado fue el PS, con 15 escaños. El PPD solo obtuvo tres y la DC uno, más un cupo independiente en su lista. El castigo a la centroizquierda podría ser consecuencia de un rechazo a los partidos tradicionales, por un lado, y una valoración crítica por no haber llevado a cabo transformaciones estructurales durante sus años en el gobierno.

A la izquierda del espectro político, la lista "Apruebo Dignidad", formada por los partidos del Frente Amplio (FA) y el Partido Comunista (PC), obtuvo 28 escaños en la Convención: siete para el PC y 21 para el FA.

La regla electoral de paridad de género para la Convención demostró que las mujeres pueden lograr un excelente desempeño electoral. Las candidatas, de hecho, superaron en votación a sus competidores masculinos, por lo que la corrección para generar paridad benefició a más hombres que mujeres. El resultado fue de 77 mujeres y 78 hombres electos. Si no se hubiera aplicado la corrección de paridad, la Convención estaría compuesta por 84 mujeres y 71 hombres. La regla sobre la integración paritaria del cuerpo constituyente llevó a que los partidos y listas apoyaran a candidatas competitivas.

Los pueblos originarios contaron, por primera vez en Chile, con 17 escaños reservados. Estos se presentaban en padrones electorales separados, y sólo podían votar personas inscritas en registros especiales para integrantes de los pueblos mapuche, aymara, diaguita, likan antay, colla, quechua, rapa nui, chango, kawashkar y yagan. El congreso no aprobó destinar un asiento a las tribus afrodescendientes.

De un electorado indígena total de más de 1,2 millones, solo el 23\% votó por estos escaños reservados. La baja participación podría deberse a la desconfianza en los procesos políticos del estado chileno, falta de información tanto de los votantes como de los encargados de realizar las votaciones, y dificultades de transporte en las zonas rurales. A pesar de la baja participación, la presencia indígena en la Convención es una señal de inclusión sin precedentes.

\section{CONCLUSIONES}

La presencia paritaria de hombres y mujeres, 17 representantes de pueblos indígenas y una constituyente con discapacidad (de 47 que compitieron gracias a una cuota legal de $5 \%$, la primera medida de acción afirmativa en Chile para este grupo) genera un nivel de inclusión nunca antes visto en un órgano representativo en Chile. Además, la Ilegada de líderes sociales que aprovecharon el sistema proporcional para unir fuerzas entre varias candidaturas con fuerte arraigo local, sin presencia en los medios de comunicación ni las redes sociales, generó un nuevo tipo de representante político. La convención constitucional tiene una composición social, etaria, étnica y de género sin precedentes, lo que resalta aún más cuán elitista y excluyente ha sido el sistema político chileno de las últimas décadas.

El proceso constituyente emanó de un movimiento social que expresa un momento constituyente; un quiebre entre normas sociales e instituciones demasiado rígidas heredadas de la dictadura. Este quiebre entre sociedad e instituciones fue canalizado por el sistema político especialmente a través de reformas aprobadas en el congreso para permitir el reemplazo constitucional. La inédita composición de la convención constitucional ofrece una apertura a grupos marginados y excluidos que puede contribuir a acortar la brecha entre sociedad e instituciones.

Existen altas expectativas en relación a los cambios que traerá el reemplazo de la Constitución de 1980 , con sus sesgos pro mercado y su mirada restrictiva de la participación, por una carta fundamental democrática. Es cierto que la constitución, por sí sola, no establecerá programas políticos o sociales para resolver las demandas implícitas en las movilizaciones sociales de la revuelta de 2019 . La constitución puede, sin embargo, fomentar políticas que permitan superar la extrema desigualdad y el elitismo político que esta carta fundamental ha impedido cambiar. Puede contribuir así a que la política electoral sea más inclusiva y legítima. También puede potenciar nuevas formas de participación ciudadana deliberativa. Con respecto a los derechos sociales y económicos, la nueva constitución podría enviar un mensaje relevante al sistema político, incluidos el congreso y los tribunales, de que se trata de una cuestión de responsabilidad colectiva en lugar de una preocupación exclusivamente individual.

El movimiento político que desencadenó el proceso constituyente no fue orquestado por ningún grupo o partido político, ni por un movimiento o líder social específico. Esta es una buena noticia para quienes temen que simplemente se busque re- 
emplazar una élite por otra, o que se pueda crear espacio para un populismo autoritario. Por el contrario, parece claro que las fuerzas que impulsaron la revuelta son variadas y plurales, con diferentes demandas que convergen en la necesidad de un acuerdo social básico verdaderamente democrático.

La pandemia de Covid 19, que en Chile se desató a comienzo de 2020, forzó a realizar varias modificaciones al calendario electoral, postergando primero el plebiscito de inicio del proceso constituyente, y luego la elección de integrantes de la convención. También llevó a la declaración de un estado constitucional de catástrofe durante un año y medio, con toques de queda nocturnos y restricciones a la circulación de las personas. El gobierno había declarado otro estado de excepción constitucional, el estado de emergencia, inmediatamente después de la revuelta de octubre de 2019. Es en este contexto que se desarrollan los debates políticos y electorales del momento constituyente chileno.

No hay duda de que la pandemia afectó el desarrollo de las protestas y la naturaleza de la participación ciudadana, que fue muy intensa en 2019. Los cabildos y reuniones locales en los barrios fueron reemplazados por transmisiones a través de redes sociales desde marzo de 2020. La pandemia también desvió, hasta cierto punto, los intereses de la ciudadanía, enfocada ahora más a superar una crisis sanitaria y económica que al debate constitucional.

El reemplazo constitucional ha sido a menudo el resultado de crisis revolucionarias o rupturas autoritarias. El proceso constituyente chileno es un intento de canalizar democráticamente un momento constituyente sin tal ruptura. En el contexto de descrédito de la política y las instituciones en el país, transitar esta delgada línea requerirá un esfuerzo extraordinario de diálogo y apertura a la inclusión de actores políticos excluidos durante mucho tiempo de la toma de decisiones. El éxito del proceso dependerá de su capacidad de construir un sistema institucional más democrático, capaz de canalizar en forma efectiva las preferencias sociales, dejando atrás el legado institucional de la dictadura.

\section{REFERENCIAS}

Ahumada, P. (2018). El Tribunal Constitucional en Chile: tres ensayos de justicia constitucional. En C. Huneeus y O. Avendaño (Eds.). El sistema político de Chile (pp. 379-410). LOM.

AtrIA, F. (2013). La Constitución tramposa. LOM.
BAROzet, E. (2016). Entre la urna, las redes sociales y la calle. Las relaciones entre movimientos sociales y partidos políticos en el Chile democrático. En M. Garretón (Ed.). La gran ruptura. Institucionalidad política y actores sociales en el Chile del siglo XXI. Santiago: LOM.

BASSA, J. (2015). El Tribunal Constitucional en la Constitución chilena vigente. En J. Bassa, J.C. Ferrada y C. Viera (Eds). La Constitución chilena. Una visión crítica a su práctica política (pp. 253-284). LOM.

Busch, T. (2012). El concepto de Constitución y la incomodidad constitucional en Chile. Global Jurist, 12(2), artículo 5. http://doi.org/10.1515/19342640.1410

Causa, O. y Hermansen, M. (2019). Income Redistribution Through Taxes and Transfers Across OECD Countries. Economics Department Working Papers No. 1453. https://doi.org/10.1108/S1049258520200000028002

COES. (2021). Resultados encuesta: percepciones y creencias de las élites económica, política y cultural de Chile. https://coes.cl/resultados-encuesta-percepciones-y-creencias-de-las-elites-economica-politica-y-cultural-de-chile/

Donoso, S. y Von Bulow, M. (2016). Social movements in Chile: Organization Trajectories, and Political Consequences. Palgrave Macmillan US.

Escudero, M. (2018). El Congreso Nacional: organización y funciones. En C. Huneeus y O. Avendaño (Eds.). El sistema político de Chile (pp. 119151). LOM.

Escudero, M. (2021). Making a Constituent Assembly Possible in Chile: The Shifting Costs of Opposing Change. Bulletin of Latin American Research. http://doi.org/10.1111/blar.13290

GarRetón, M. (2003). Incomplete Democracy. University of North Carolina Press.

Garretón, M. (2021). Política y movimientos sociales en Chile. Antecedentes y proyecciones del estallido social de octubre 2019. LOM

Heiss, C. y Szmulewicz, E. (2018). La Constitución política de 1980. En C. Huneeus y O. Avendaño, El sistema político de Chile (pp. 57-83). LOM.

Huber, E., Pribble, J. y Stephens, J. (2010). The Chilean Left in Power: Achievements, Failures, and Omissions. En K. Weyland, R. Madrid y W. Hunter (Eds.), Leftist Governments in Latin America. Successes and Shortcomings (pp. 77-97). Cambridge University Press. https://doi.org/10.1017/ CB09780511778742.004

Huneeus, C. (2016). El régimen de Pinochet. Taurus. LunA, J. y Altman, D. (2011). Uprooted but Stable: Chi- 
lean Parties and the Concept of Party System Institutionalization. Latin American Politics and Society, 53(2), 1-28. https://doi.org/10.1111/ j.1548-2456.2011.00115.x

LunA, J. (2016). Chile's Crisis of Representation. Journal of Democracy, 27(3), 129-138. https://doi. org/10.1353/jod.2016.0046

LUNA, J. (2021). ¿Es posible la articulación entre movimientos sociales y partidos políticos en el mundo contemporáneo? En M. Garretón (Ed.). Política y movimientos sociales en Chile. Antecedentes $y$ proyecciones del estallido social de octubre 2019. LOM.

Madariaga, A. (2019). La Continuidad del Neoliberalismo en Chile: ideas, instituciones e intereses. Revista de Estudos e Pesquisas sobre as Américas, 13(2). https://doi.org/10.21057/10.21057/repamv13n2.2019.23217

Moulian, T. (1997). Chile actual: anatomía de un mito. LOM.

OECD. Income Inequality. https://data.oecd.org/inequality/income-inequality.htm

PNUD Chile. (2015). Desarrollo humano en Chile. Los tiempos de la politización.

PNUD Chile. (2017). Diagnóstico sobre la participación electoral en Chile.

Segovia, C. (2015). Confianza en instituciones políticas en Chile. Documento de Referencia No.25, Espacio Público.

Somma, N., Bargsted, M. Disı R. y Medel R. (2021). No water in the oasis: the Chilean Spring of 20192020. Social Movement Studies, 20(4). http:// doi.org/10.1080/14742837.2020.1727737 